\title{
Implant Electrical Characteristics Predict Response to Cardiac Resynchronization
} Therapy

\author{
Tina Lin', Paula Crosby ${ }^{1,2}$, Hariharan Sugumar'1, Ryan Spencer ${ }^{1}$, \\ Michael Darragh Flannery', David 0'Donnell1,2 \\ ${ }^{1}$ Department of Electrophysiology, Austin Health, Heidelberg, Australia \\ ${ }^{2}$ Heart Care Victoria, Heidelberg, Australia \\ Email: tina9989@hotmail.com
}

Received 24 July 2014; revised 23 August 2014; accepted 2 September 2014

Copyright (C) 2014 by authors and Scientific Research Publishing Inc.

This work is licensed under the Creative Commons Attribution International License (CC BY). http://creativecommons.org/licenses/by/4.0/

(c) (i) Open Access

\section{Abstract}

Background: The optimal site for left ventricular (LV) lead placement in cardiac resynchronization therapy (CRT) remains uncertain. Intra-procedural measures for predicting response to CRT have shown mixed results. Hypothesis: This study analyzed intracardiac electrogram (IEGM) characteristics at implant and assessed patients' response rates (RR) to CRT. Methods: Forty-one consecutive patients undergoing CRT were enrolled. Medically optimized patients in sinus rhythm, with ejection fraction (EF) $<35 \%$, and a dyssynchrony index (DI) $>34$ were included. Right ventricular (RV) leads were positioned mid-septum. LV leads were targeted to the latest mechanical activation on echocardiography. IEGMs were measured, assessing intrinsic RV-to-LV delay (int RV-LV), RVpaced delay (RVp-LV), and LV-paced delay (LVp-RV). The difference between LVp-RV and RVp-LV was recorded as delta-LV. Response was defined as improvement of $\mathrm{EF}>10 \%$, reduction in LVEDD $>15 \%$ and improvement of $\geq 1$ NYHA class. Results: Overall RR was $79 \%$. LV leads were placed in the target location in 91\%. Int RV-LV was $101 \pm 14 \mathrm{~ms}$ in responders; $78 \pm 11 \mathrm{~ms}$ in nonresponders $(p<0.05)$. Int RV-LV > 100 had a RR of $87 \%$; int RV-LV < 100 had a RR of $68 \%$. LVp-RV and RVp-LV did not differ significantly between responders and non-responders. Delta-LV $>40 \mathrm{~ms}$ had a RR of 56\%; delta-LV $<40 \mathrm{~ms}$ had a RR of $85 \%$. There was no significant correlation between lead position, DI, QRS duration or EF and IEGM measurements. Conclusions: IEGM measures at implant are easily obtained. Significant intrinsic electrical delay and shorter delta-LV both predict response, even when LV leads are implanted in the targeted mechanically-delayed segment. These assessments of electrical dyssynchrony may be used to determine optimal lead positions and response to CRT.

\section{Keywords}

Cardiac Resynchronization Therapy, Biventricular Pacing, Intracardiac Electrograms, Pacemaker

How to cite this paper: Lin, T., Crosby, P., Sugumar, H., Spencer, R., Flannery, M.D. and O'Donnell, D. (2014) Implant Electrical Characteristics Predict Response to Cardiac Resynchronization Therapy. World Journal of Cardiovascular Diseases, 4, 513-521. http://dx.doi.org/10.4236/wjcd.2014.41062 


\section{Introduction}

Many multicenter, randomized clinical trials have demonstrated that cardiac resynchronization therapy (CRT) can improve outcomes in the majority of patients with left ventricular (LV) systolic dysfunction and wide QRS complex [1]-[4]. CRT improves heart failure symptoms and reduces hospitalizations and mortality. However, approximately $30 \%$ of patients do not respond to treatment [1]-[4], and the degree of improvement is not uniform. Many studies have therefore been conducted in an attempt to define factors that could improve overall RR [5] [6].

The implant location of the LV lead has been shown to affect mechanical dyssynchrony, response to CRT and overall mortality [7]. Therefore, mechanical dyssynchrony has so far been the treatment target for CRT, and QRS prolongation indicating electrical dyssynchrony has been used as a surrogate for mechanical dyssynchrony [8]-[12]. Electrical and mechanical dyssynchrony in individuals often correlate, suggesting that baseline electrical characteristics could help predict response to CRT. Although transthoracic echocardiography (TTE) can be used to assist in identifying the area of latest mechanical activation pre-procedure, methods to assess for and find the optimal LV lead position intra-procedurally have had mixed results [13]-[15]. Several studies have measured QRS to LV intracardiac electrogram (IEGM) delays (QLV) and found reasonable correlations with response to CRT [16]. Finding a simple, intra-procedural measure that can be easily and repeatedly assessed will allow the LV lead to be repositioned to the optimal position during the index procedure.

The objectives of this study were to: 1 ) investigate LV myocardial conduction characteristics using IEGMs of the right ventricular (RV) and LV leads at the time of CRT implantation; 2) assess the correlation of these measures of electrical dyssynchrony with mechanical dyssynchrony; and 3) determine which electrical parameters result in improved RR and therefore predict response to CRT.

\section{Methods}

This was a prospective, single centre study of consecutive patients scheduled for CRT implantation according to current guidelines [17]. Specifically, patients were accepted for CRT after a period of medical optimization, all had symptomatic heart failure (NYHA Class II-IV) and left ventricular ejection fractions (LVEF) < 35\%. Only patients in sinus rhythm (SR), with LBBB and QRS duration $\geq 120 \mathrm{~ms}$, and dyssynchrony index (DI) $>34$ on TTE were enrolled.

Prior to CRT implantation all patients underwent baseline 12-lead ECG, TTE and assessment of NYHA functional class. All patients provided informed consent.

\subsection{Echocardiography}

The echocardiographic examination was performed using the Vivid 7 \& 9 (General Electric, WI, USA). LV enddiastolic volume (LVEDV), LV end-systolic volume (LVESV), and LVEF were assessed using the modified Simpson's biplane method. Interventricular mechanical dyssynchrony (IVMD) was assessed as the difference between the QRS to onset of pulmonary artery flow (QRS-PA) and QRS to onset of aortic flow (QRS-Ao), and dyssynchrony was significant if the IVMD was $>40 \mathrm{~ms}$. Intraventricular dyssynchrony was assessed by 2-dimensional color tissue Doppler imaging (TDI) in the 4-chamber, 2-chamber and 3-chamber apical views, and mechanical velocity curves were analyzed. Significant dyssynchrony was defined as a DI $>34$, as previously described [18]-[21].

\subsection{CRT Implantation Procedure}

Transvenous CRT implantation was performed under sedation and local anesthesia. The RV defibrillation lead was positioned in the mid septum defined by fluoroscopic imaging in the anterior-posterior (AP) and left anterior oblique (LAO) $30^{\circ}$ views. The right atrial (RA) lead was positioned in the RA septum or RA appendage. After venography of the coronary vein tributaries, the LV lead was targeted to the segment of latest mechanical 
activation identified on pre-procedure echocardiographic TDI. Bipolar and quadripolar leads were both used in this analysis.

\subsection{Intracardiac Electrical Measures}

Intracardiac EGMs were assessed using the pacing programmer of respective device companies. The intrinsic electrical delay (int RV-LV) was measured during intrinsic rhythm from the initial EGM deflection in the RV lead to the earliest peak of the local unipolar signal on each electrode of the LV lead (Figure 1). The RV-paced delay (RVp-LV) (Figure 2) and LV-paced delay (LVp-RV) (Figure 3) were measured during RV-only pacing

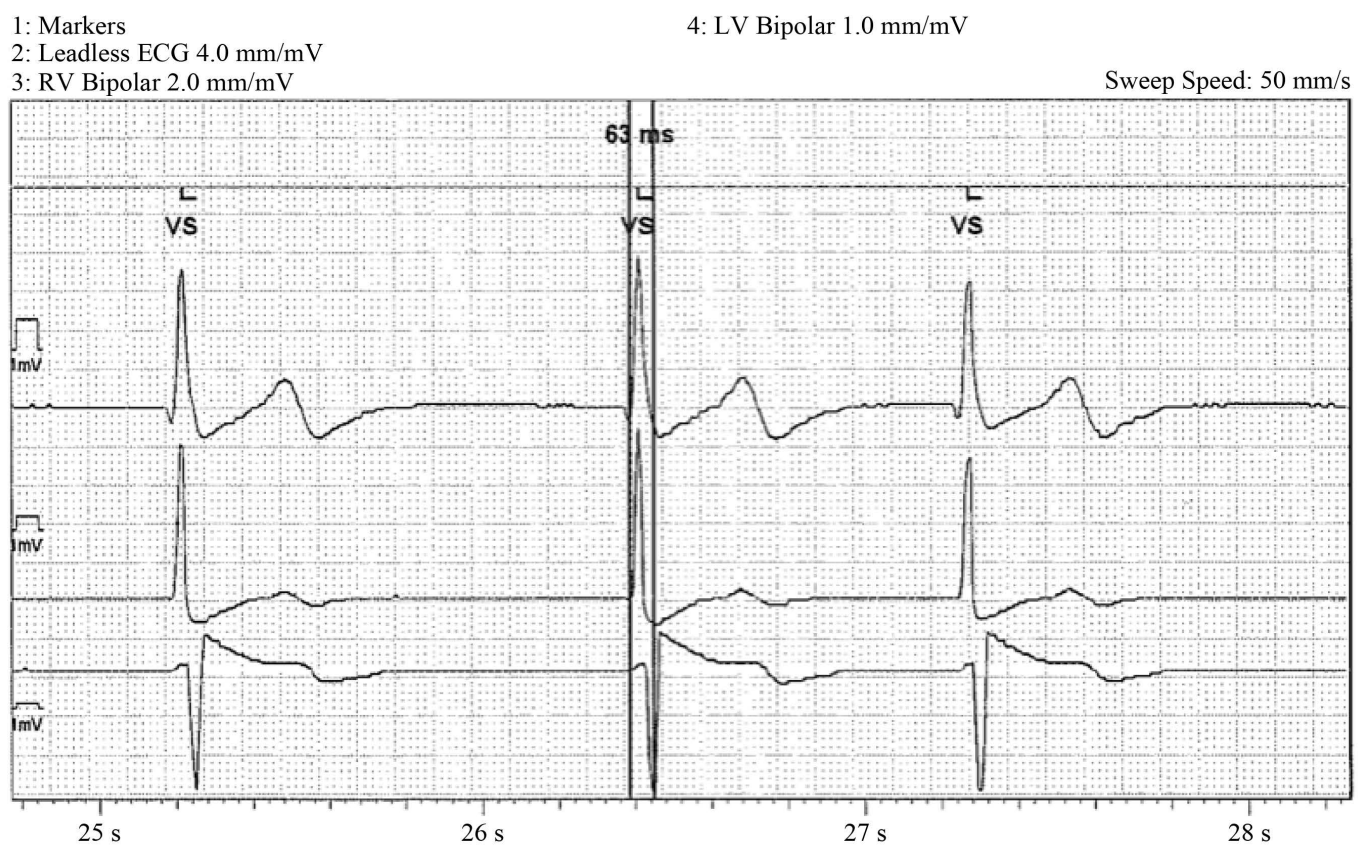

Figure 1. Intracardiac electrograms-int RV-LV.

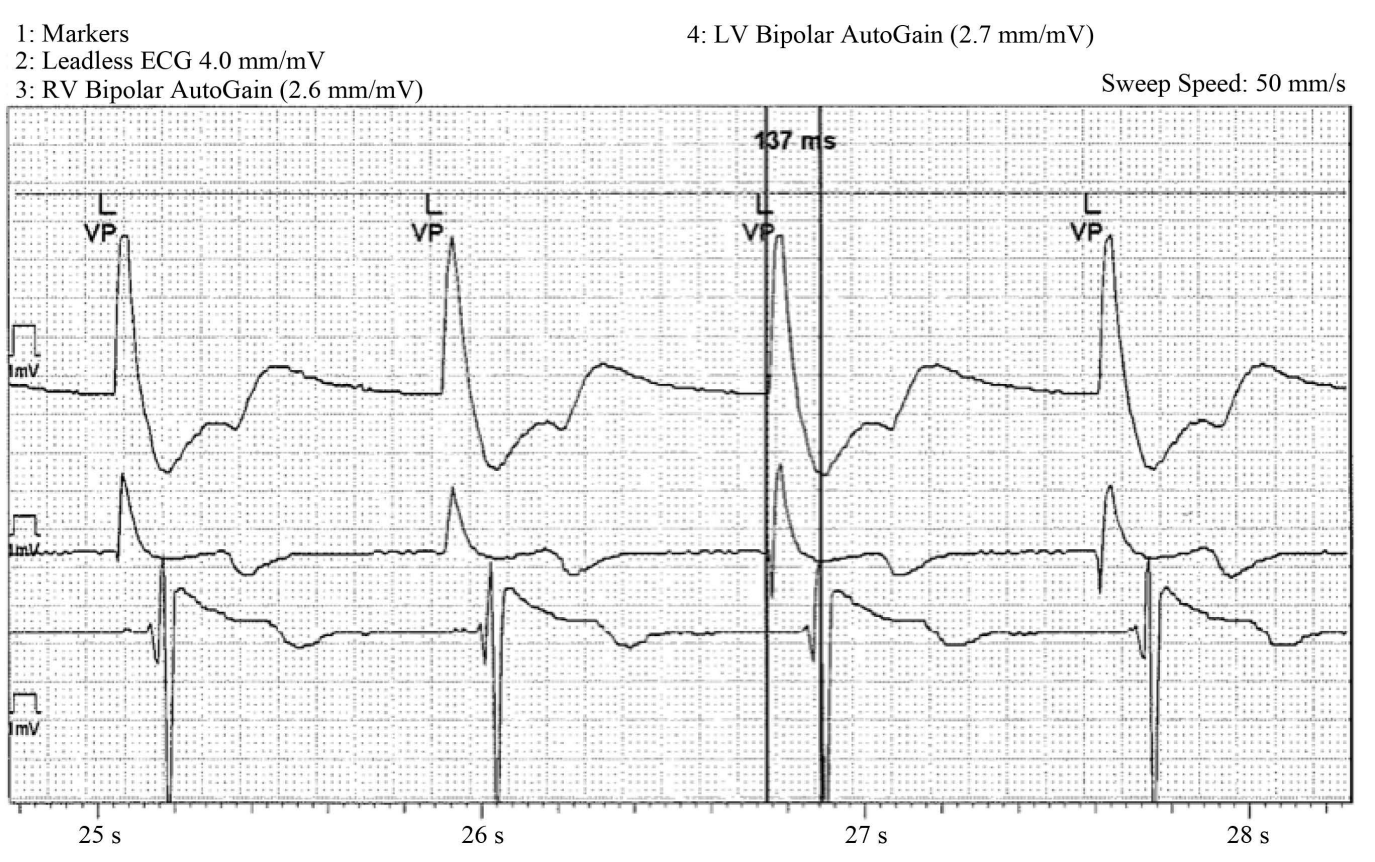

Figure 2. Intracardiac electrograms-RVp-LV. 


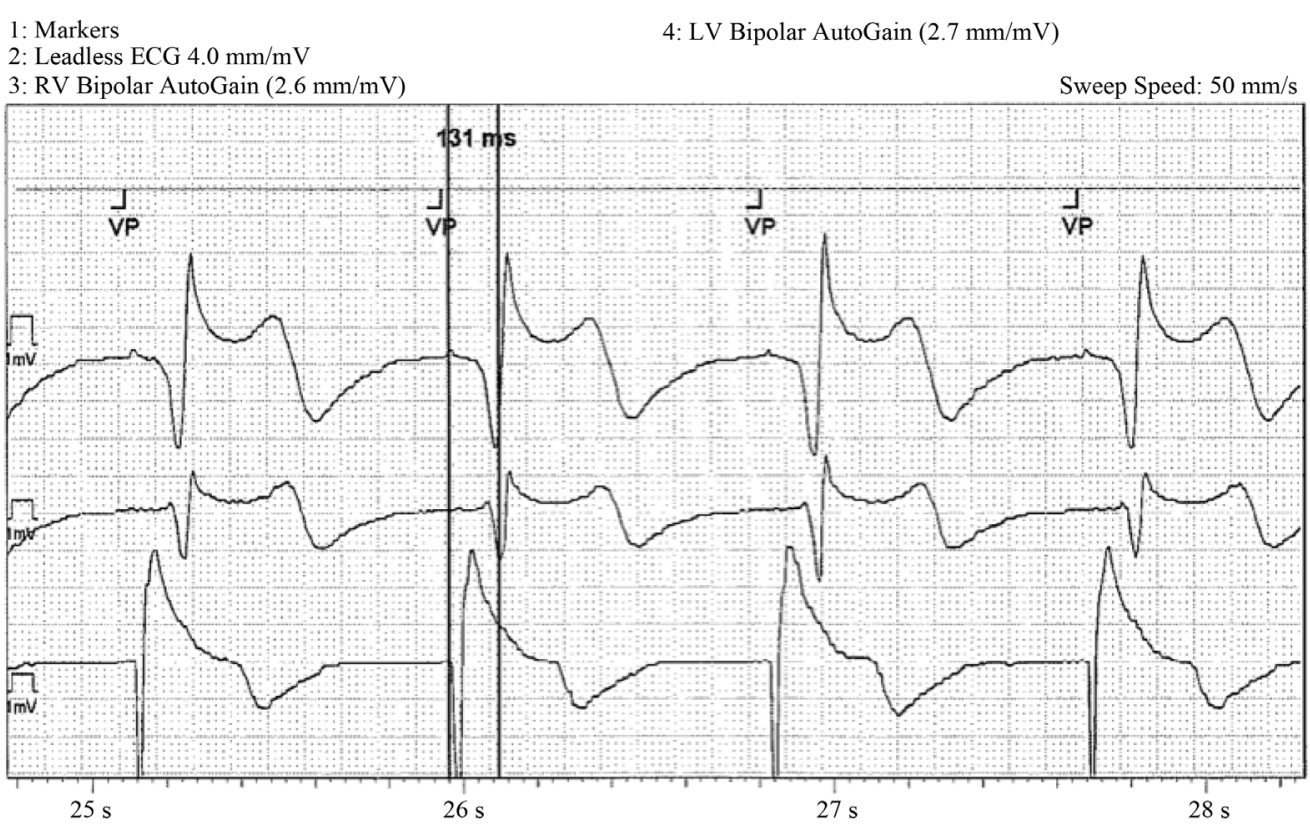

Figure 3. Intracardiac electrograms-LVp-RV. Delta LV is calculated as LVp-RV - RVp-LV.

and LV-only pacing respectively. The timing difference between the LVp-RV and RVp-LV was calculated as the delta LV. Pacing was performed with an output two-times the measured threshold at a rate of 70 beats/min or 10 beats/min above the patient's intrinsic heart rate.

The absolute QLV interval was measured in SR as the interval from the QRS onset to the first large positive or negative peak of the LV IEGM. Percentage QLV (\% QLV) was calculated as the absolute QLV/QRS duration.

According to our unit protocol, all LV leads were successfully repositioned if the int RV-LV electrical delay was $<60 \mathrm{~ms}$ [13]-[15].

\subsection{Post Implant Procedure}

All patients were programmed following CRT implantation to biventricular pacing with AV and VV delays programmed according to QuickOpt ${ }^{\mathrm{TM}}$ (St Jude Medical, MN, USA) calculations. The specific LV electrode used for pacing was at the discretion of the implanting electrophysiologist and this was maintained until at least the 3-month visit. Data analysis was performed using only the electrode delivering chronic LV pacing. Device interrogation was performed at 24 hours, 2, 6 and 12 weeks to ensure maximal delivery of CRT.

\subsection{Response to CRT}

At 3-month follow-up IEGMs, surface 12-lead ECGs and TTE measures were evaluated. Response to CRT was defined as a combined end-point of improvement in LVEF > 10\%, reduction in LV end-diastolic diameter (LVEDD) $>15 \%$ and symptomatic improvement of at least 1 NYHA functional class.

\subsection{Data and Statistical Analysis}

The results were presented as mean \pm SD. Statistical significance was defined as $p<0.05$. Paired and unpaired t-tests and Fishers exact test were used when appropriate. Receiver operating curves analysis was performed to predict effectiveness of individual variables.

\section{Results}

Sixty-nine consecutive patients undergoing initial CRT implantation were screened and 44 patients meeting criteria were prospectively enrolled. 10 patients were excluded because of atrial fibrillation (AF), 7 because of QRS duration $\leq 120 \mathrm{~ms}, 5$ due to absence of dyssynchrony on TDI, and 3 due to RBBB. Forty-one (93\%) pa- 
tients completed the 3-month follow-up. One patient did not complete follow-up due to LV lead displacement, 1 due to non-cardiac death, and 1 withdrew due to relocation. Baseline characteristics are shown in Table 1.

All patients had successful LV lead placement, with 93\% (38/41) of LV leads placed in the pre-identified target segment of latest mechanical activation. The target segment was the lateral, posterolateral, or anterolateral segment in 38 patients, the apex in 2 patients and a true anterior segment in 1 patient.

At 3-months follow-up, a mean of 99.1\% biventricular pacing was achieved, calculated by the implanted device. The mean programmed AV delay was $131 \pm 21$ ms and mean VV delay was an LV predominance of $27 \pm$ $13 \mathrm{~ms}$.

32 patients ( $\mathrm{RR}=79 \%)$ were defined as responders to CRT at 3 months. There was a mean EF improvement of $11 \%$ and mean reduction in LVEDD of $16 \%$.

The int RV-LV at implant was longer in responders (101 $\pm 14 \mathrm{~ms})$ compared to non-responders (78 $\pm 11 \mathrm{~ms})$, $p<0.05$. The RVp-LV was $112 \pm 15$ in responders and $102 \pm 17$ in non-responders ( $p=$ NS). The LVp-RV was $138 \pm 20 \mathrm{~ms}$ in responders and $149 \pm 17 \mathrm{~ms}$ in non-responders $(p=\mathrm{NS})$. The delta LV was shorter in responders ( $21 \pm 17 \mathrm{~ms}$ ) than in non-responders ( $51 \pm 10 \mathrm{~ms}), p<0.05$.

ROC analysis was used to determine RR for the IEGM characteristics. Patients with int RV-LV $>100 \mathrm{~ms}$ had a RR of $87 \%$, while those with int RV-LV $<100 \mathrm{~ms}$ had a RR of $68 \%$ (Figure 4). A delta LV $>40 \mathrm{~ms}$ was associated with a RR of 56\%, compared with a delta $\mathrm{LV}<40 \mathrm{~ms}$ ( $\mathrm{RR}=85 \%$ ) (Figure 5).

Table 1. Baseline patient characteristics.

\begin{tabular}{cccc}
\hline & Baseline & At 3 months & Change \\
\hline Age & $63.1 \pm 11.2$ & & \\
Sex male (\%) & $76 \%$ & & \\
Ischemic (\%) & $49 \%$ & & \\
QRS duration (ms) & 153.4 & & $11.4 \%$ \\
LVEF (\%) & 23.4 & 34.9 & $12.8 \%$ \\
LVEDD (mm) & 66.2 & 57.7 & $12.1 \%$ \\
TDI & 46.4 & 34.2 & $13.9 \%$ \\
Int RV-LV (ms) & 96.5 & 82.6 & $13.8 \%$ \\
RVp-LV (ms) & 111.0 & 105.7 & $23.6 \%$ \\
LVp-RV (ms) & 139.4 & 122.4 & \\
\hline
\end{tabular}

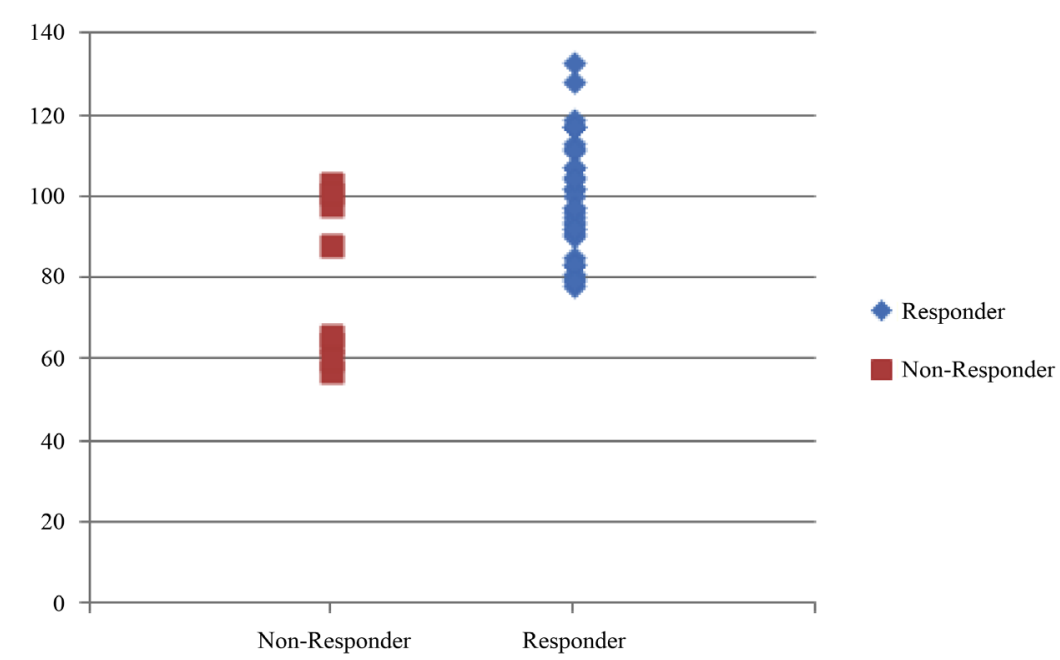

Figure 4. Int RV-LV in responders vs non-responders. An int RV-LV $>100 \mathrm{~ms}$ had a RR of $87 \%$. An int RV-LV $<100$ ms had a RR of $68 \%$. 


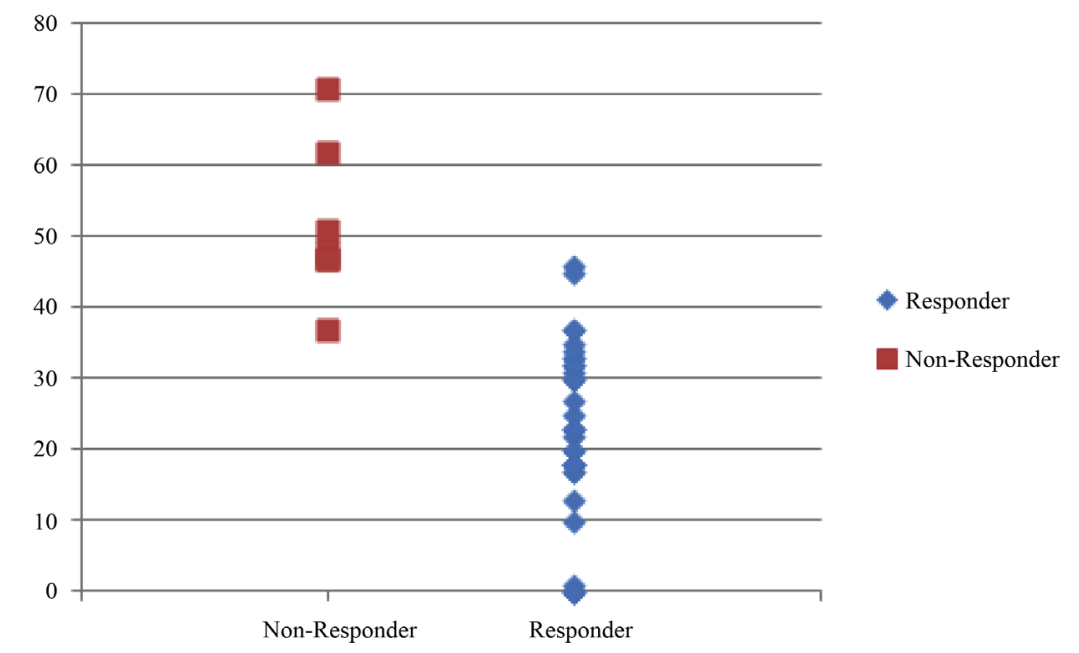

Figure 5. Delta LV in responders vs non-responders. A delta LV $>40 \mathrm{~ms}$ had a RR of $56 \%$. A delta $\mathrm{LV}<40 \mathrm{~ms}$ had a RR of $85 \%$.

QLV was $127 \pm 14 \mathrm{~ms}$ (80\%) in responders and $116 \pm 15 \mathrm{~ms}$ (73\%) in non-responders ( $p=\mathrm{NS})$. QLV was $125 \pm$ $14 \mathrm{~ms}$ (80\%) when the LV lead was placed in the target location and $100 \pm 7 \mathrm{~ms}(63 \%)$ when in non-target locations. The previously defined QLV > 75\% was associated with a RR of $89 \%$ and QLV $<75 \%$ had a RR of $61 \%$ [22]. The correlation between QLV and int RV-LV was modest $\left(r^{2}=0.34\right)$.

There was no significant difference in baseline QRS duration, LVEF or DI between responders and non-responders. There was no significant correlation between baseline measures of QRS duration LVEF, DI or LV lead position and the IEGM measurements.

\section{Discussion}

This prospective study demonstrated that IEGM measurements obtained at implant can predict response to therapy in patients undergoing CRT implantation. There was a correlation between both the EGM measures of intrinsic RV-LV EGM delay (int RV-LV) and delta LV, and response to CRT at 3 months.

CRT has been shown to improve LV systolic function in a significant number of patients with systolic heart failure. In the last decade, a number of randomized, multicenter studies have demonstrated that CRT reduces overall morbidity and mortality, and have established CRT as a fundamental part of the management of LV systolic heart failure [1]-[4]. Despite an overall improvement in cardiac parameters, response to CRT is not uniform, with a documented non-response rate between $20 \%$ - 40\% [4] [6]. Many factors have been postulated to explain such a significant non-response rate, and the LV-lead position is consistently listed as a variable impacting on RR [23] [24]. Attempts to place the LV-lead at the site of latest of mechanical activation have generally yielded positive results in predicting response [25]-[28], however data remains at best predictive in a population sense. The ability to identify the latest area of mechanical activation is also limited by image quality and reproducibility issues with TTE and other imaging modalities such as gadolinium enhanced MRI [29] [30]. These modalities are also performed pre-procedurally, making it difficult to confirm optimal LV lead position during implantation.

Electrical measures to predict response are appealing as they are easy to perform. In addition, they allow intra-operative assessment of lead location. Acute QRS narrowing with biventricular pacing is poorly predictive of response over time [31]. Various measures of surface ECG to intracardiac LV EGMs, in particular QLV measurements, have shown strong association with reverse remodelling with CRT [22] [32]. The QLV measure in part represents an attempt to place the lead in an electrically delayed segment of the LV. This study has used an alternative measure of electrical delay, and results support that of previous publications which demonstrated that LV leads placed at areas of electrical delay are more likely to result in response to CRT.

The IEGM measures used in this study incorporate both RV and LV leads. The majority of patients with CRT are paced from both ventricular leads and the interaction between the two leads would logically influence response [33]. This result is consistent with several recent studies assessing various other IEGM measures, all of which have correlated longer RV-LV intervals to response to CRT [13]-[15]. A unique aspect of this analysis was 
the high rate (93\%) of LV-lead placement in the target location of latest mechanical activation. This is reflected in the results, where a mean int RV-LV of 96 ms was obtained, as compared to previous analyses (mean int RV-LV = $60 \mathrm{~ms}$ ) [13]-[15]. In addition, the mean \%QLV of 79\% and a QLV > $95 \mathrm{~ms}$ in 93\% of our study population is significantly higher than previous analyses (\%QLV $=55 \%)$. The data from our analysis adds to the previous work confirming that IEGM measures are useful in predicting response to CRT and confirms that the int RV-LV measure is equally valid even when the LV lead is placed at the targeted zone of latest mechanical activation.

The second and novel finding was that a significant delta LV was associated with poor response to CRT. The delta LV in essence measures the difference in the paced electrical activation from the RV and LV leads. In a heart with homogenous ventricular electrical conduction, the delta LV should be zero. An increasing delta LV reflects slower electrical conduction during LV versus RV-pacing. This most likely represents absolute or functional conduction delay from LV-pacing, and we postulate that the poor response obtained with higher delta LVs reflects LV-pacing in or adjacent to areas of significant myocardial scarring. Further analysis to correlate delta LV and scar using MRI would be valuable. The alternative explanation is that during RV pacing, RV electrical activation is more rapid secondary to recruitment of the specialized conducting system. However, the ability to incorporate the specialized conducting tissue should improve response to CRT, and given our results were contradictory this seems less likely. Other publications that have assessed measures similar to delta LV have reported much lower delta LVs and often delta LV values close to zero.

\section{QLV vs EGM}

Previous data on the value of QLV to predict response to CRT was confirmed during this study. Whilst mean QLV did not differ significantly between responders and non-responders, a QLV > 75\% produced a similar RR to previous studies. The int RV-LV and QLV are different measures of electrical dyssynchrony. The use of RV to LV EGMs incorporates both ventricular leads and underpins our belief that the relative positions and electrical parameters of both leads are important in CRT response. The finding that the intrinsic EGM delays were significantly better at predicting response to CRT than the RV paced delays was unexpected. Given that all patients were paced close to $100 \%$ of the time from the RV lead, we expected this value to be as predictive as int RV-LV in predicting response. In our study, the RV lead was positioned in the mid-septum and different RV lead positions may yield different findings.

\section{Limitations}

These data represent a single centre study and the findings overlap that of previous studies measuring QLV. The high percentage of LV leads positioned at zones of latest mechanical activation and our unit policy to reposition all leads to sites with int RV-LV > 60 ms could potentially bias the data. These results however, confirm and emphasize the value of electrical measures to predict response even at "optimal" anatomical locations.

The study population was a carefully selected, relatively homogenous group with strict inclusion criteria, and caution is needed before extrapolating these results to the CRT population as a whole.

Prolonged int RV-LV is a marker of the electrical conduction abnormalities in heart failure patients and prolonged delta LV is a marker of electrical heterogeneity, and whilst these parameters predict response to CRT the mechanistic effects postulated have not yet been proven.

\section{Conclusion}

This study demonstrates that IEGM measurements at implant can be easily obtained intra-procedurally using available pacing parameters. Significant int RV-LV delay and homogenous conduction measured as a shorter delta LV, both predicted response to CRT. These electrical measures predict response even with LV leads placed at target sites of latest mechanical activation. These assessments of electrical dyssynchrony may be used during implant to guide LV lead positioning, and predict response to CRT.

\section{References}

[1] Abraham, W.T., Fisher, W.G. and Smith, A.L. (2002) Cardiac Resynchronization in Chronic Heart Failure. The New England Journal of Medicine, 346, 1845-1853. http://dx.doi.org/10.1056/NEJMoa013168 
[2] Young, J.B., Abraham, W.T. and Smith, A.L. (2003) Combined Cardiac Resynchronization and Implantable Cardioversion Defibrillation in Advanced Chronic Heart Failure: The MIRACLE ICD Trial. Journal of the American Medical Association, 289, 2685-2694. http://dx.doi.org/10.1001/jama.289.20.2685

[3] Bristow, M.R., Saxon, L.A., Boehmer, J., Krueger, S., Kass, D.A., De Marco, T., et al. (2004) Cardiac-Resynchronization Therapy with or without an Implantable Defibrillator in Advanced Chronic Heart Failure. The New England Journal of Medicine, 350, 2140-2150. http://dx.doi.org/10.1056/NEJMoa032423

[4] Cleland, J.G.F., Daubert, J.-C., Erdmann, E., Freemantle, N., Gras, D., Kappenberger, L., et al. (2005) The Effect of Cardiac Resynchronization on Morbidity and Mortality in Heart Failure. The New England Journal of Medicine, 352, 1539-1549. http://dx.doi.org/10.1056/NEJMoa050496

[5] Chung, E.S., Leon, A.R., Tavazzi, L., Sun, J.-P., Nihoyannopoulos, P., Merlino, J., et al. (2008) Results of the Predictors of Response to CRT (PROSPECT) trial. Circulation, 117, 2608-2616. http://dx.doi.org/10.1161/CIRCULATIONAHA.107.743120

[6] Moss, A.J., Hall, W.J., Cannom, D.S., Klein, H., Brown, M.W., Daubert, J.P., et al. (2009) Cardiac-Resynchronization Therapy for the Prevention of Heart-Failure Events. The New England Journal of Medicine, 361, 1329-1338. http://dx.doi.org/10.1056/NEJMoa0906431

[7] Dong, Y.-X., Powell, B.D., Asirvatham, S.J., Friedman, P.A., Rea, R.F., Webster, T.L., et al. (2012) Left Ventricular Lead Position for Cardiac Resynchronization: A Comprehensive Cinegraphic, Echocardiographic, Clinical, and Survival Analysis. Europace, 14, 1139-1147. http://dx.doi.org/10.1093/europace/eus045

[8] McMurray, J.J.V., Adamopoulos, S., Anker, S.D., Auricchio, A., Böhm, M., Dickstein, K., et al. (2012) ESC Guidelines for the Diagnosis and Treatment of Acute and Chronic Heart Failure 2012: The Task Force for the Diagnosis and Treatment of Acute and Chronic Heart Failure 2012 of the European Society of Cardiology. Developed in Collaboration with the Heart Failure Association (HFA) of the ESC. European Heart Journal, 33, 1787-1847.

[9] Bax, J.J., Bleeker, G.B., Marwick, T.H., Molhoek, S.G., Boersma, E., Steendijk, P., et al. (2004) Left Ventricular Dyssynchrony Predicts Response and Prognosis after Cardiac Resynchronization Therapy. Journal of the American College of Cardiology, 44, 1834-1840. http://dx.doi.org/10.1016/j.jacc.2004.08.016

[10] Gorcsan, J., Oyenuga, O., Habib, P.J., Tanaka, H., Adelstein, E.C., Hara, H., et al. (2010) Relationship of Echocardiographic Dyssynchrony to Long-Term Survival after Cardiac Resynchronization Therapy. Circulation, 122, 1910-1918. http://dx.doi.org/10.1161/CIRCULATIONAHA.110.954768

[11] Tanaka, H., Nesser, H.J., Buck, T., Oyenuga, O., Jánosi, R.A., Winter, S., et al. (2010) Dyssynchrony by SpeckleTracking Echocardiography and Response to Cardiac Resynchronization Therapy: Results of the Speckle Tracking and Resynchronization (STAR) Study. European Heart Journal, 31, 1690-1700. http://dx.doi.org/10.1093/eurheartj/ehq213

[12] Hara, H., Oyenuga, O.A., Tanaka, H., Adelstein, E.C., Onishi, T., McNamara, D.M., et al. (2012) The Relationship of QRS Morphology and Mechanical Dyssynchrony to Long-Term Outcome Following Cardiac Resynchronization Therapy. European Heart Journal, 33, 2680-2691. http://dx.doi.org/10.1093/eurheartj/ehs013

[13] Sassone, B., Gabrieli, L., Saccà, S., Boggian, G., Fusco, A., Pratola, C., et al. (2010) Value of Right Ventricular-Left Ventricular Interlead Electrical Delay to Predict Reverse Remodelling in Cardiac Resynchronization Therapy: The Inter-V Pilot Study. Europace, 12, 78-83. http://dx.doi.org/10.1093/europace/eup347

[14] Zucchelli, G., Soldati, E., Di Cori, A., De Lucia, R., Segreti, L., Solarino, G., et al. (2010) Role of Intraoperative Electrical Parameters in Predicting Reverse Remodelling after Cardiac Resynchronization Therapy and Correlation with Interventricular Mechanical Dyssynchrony. Europace, 12, 1453-1459. http://dx.doi.org/10.1093/europace/euq267

[15] Kristiansen, H.M., Hovstad, T., Vollan, G., Keilegavlen, H. and Faerestrand, S. (2012) Clinical Implication of Right Ventricular to Left Ventricular Interlead Sensed Electrical Delay in Cardiac Resynchronization Therapy. Europace, 14, 986-993. http://dx.doi.org/10.1093/europace/eur429

[16] Singh, J.P., Fan, D., Heist, E.K., Alabiad, C.R., Taub, C., Reddy, V., et al. (2006) Left Ventricular Lead Electrical Delay Predicts Response to Cardiac Resynchronization Therapy. Heart Rhythm, 3, 1285-1292. http://dx.doi.org/10.1016/j.hrthm.2006.07.034

[17] Russo, A.M., Stainback, R.F., Bailey, S.R., Epstein, A.E., Heidenreich, P.A., Jessup, M., et al. (2013) ACCF/HRS/ AHA/ASE/HFSA/ SCAI/SCCT/SCMR 2013 Appropriate Use Criteria for Implantable Cardioverter-Defibrillators and Cardiac Resynchronization Therapy: A Report of the American College of Cardiology Foundation Appropriate Use Criteria Task Force, Heart Rhythm Society, American Heart Association, American Society of Echocardiography, Heart Failure Society of America, Society for Cardiovascular Angiography and Interventions, Society of Cardiovascular Computed Tomography, and Society for Cardiovascular Magnetic Resonance. Heart Rhythm, 10, e11-e58.

[18] Yu, C.M., Fung, J.W.H., Zhang, Q., Chan, C.K., Chan, Y.S., Lin, H., et al. (2004) Tissue Doppler Imaging Is Superior to Strain Rate Imaging and Postsystolic Shortening on the Prediction of Reverse Remodeling in both Ischemic and Nonischemic Heart Failure after Cardiac Resynchronization Therapy. Circulation, 110, 66-73.

http://dx.doi.org/10.1161/01.CIR.0000133276.45198.A5 
[19] Kearney, L.G., Wai, B., Ord, M., Burrell, L.M., O’Donnell, D. and Srivastava, P.M. (2011) Validation of Rapid Automated Tissue Synchronization Imaging for the Assessment of Cardiac Dyssynchrony in Sinus and Non-Sinus Rhythm. Europace, 13, 270-276. http://dx.doi.org/10.1093/europace/euq442

[20] Wai, B., Kearney, L.G., Burrell, L.M., Ord, M. and Srivastava, P.M. (2012) Normalization of Ejection Fraction in Subjects with Systolic Heart Failure. Is It Really Normal? A Myocardial Deformation Study. International Journal of Cardiology, 154, 365-367. http://dx.doi.org/10.1016/j.ijcard.2011.11.036

[21] Kearney, L.G., Lu, K., Ord, M., Patel, S.K., Profitis, K., Matalanis, G., et al. (2012) Global Longitudinal Strain Is a Strong Independent Predictor of All-Cause Mortality in Patients with Aortic Stenosis. European Heart Journal, Cardiovascular Imaging, 13, 827-833. http://dx.doi.org/10.1093/ehjci/jes115

[22] Gold, M.R., Birgersdotter-Green, U., Singh, J.P., Ellenbogen, K.A., Yu, Y., Meyer, T.E., et al. (2011) The Relationship between Ventricular Electrical Delay and Left Ventricular Remodelling with Cardiac Resynchronization Therapy. European Heart Journal, 32, 2516-2524. http://dx.doi.org/10.1093/eurheartj/ehr329

[23] Díaz-Infante, E., Mont, L., Leal, J., García-Bolao, I., Fernández-Lozano, I., Hernández-Madrid, A., et al. (2005) Predictors of Lack of Response to Resynchronization Therapy. The American Journal of Cardiology, 95, 1436-1440.

[24] Ruschitzka, F., Abraham, W.T., Singh, J.P., Bax, J.J., Borer, J.S., Brugada, J., et al. (2013) Cardiac-Resynchronization Therapy in Heart Failure with a Narrow QRS Complex. The New England Journal of Medicine, 369, 1395-1405. http://dx.doi.org/10.1056/NEJMoa1306687

[25] Saba, S., Marek, J., Schwartzman, D., Jain, S., Adelstein, E., White, P., et al. (2013) Echocardiography-Guided Left Ventricular Lead Placement for Cardiac Resynchronization Therapy: Results of the Speckle Tracking Assisted Resynchronization Therapy for Electrode Region Trial. Circulation: Heart Failure, 6, 427-434. http://dx.doi.org/10.1161/CIRCHEARTFAILURE.112.000078

[26] Khan, F.Z., Virdee, M.S., Palmer, C.R., Pugh, P.J., O’Halloran, D., Elsik, M., et al. (2012) Targeted Left Ventricular Lead Placement to Guide Cardiac Resynchronization Therapy: The TARGET Study: A Randomized, Controlled Trial. Journal of the American College of Cardiology, 59, 1509-1518. http://dx.doi.org/10.1016/j.jacc.2011.12.030

[27] Wong, J.A., Yee, R., Stirrat, J., Scholl, D., Krahn, A.D., Gula, L.J., et al. (2013) Influence of Pacing Site Characteristics on Response to Cardiac Resynchronization Therapy. Circulation: Cardiovascular Imaging, 6, 542-550. http://dx.doi.org/10.1161/CIRCIMAGING.111.000146

[28] Döring, M., Braunschweig, F., Eitel, C., Gaspar, T., Wetzel, U., Nitsche, B., et al. (2013) Individually Tailored Left Ventricular Lead Placement: Lessons from Multimodality Integration between Three-Dimensional Echocardiography and Coronary Sinus Angiogram. Europace, 15, 718-727. http://dx.doi.org/10.1093/europace/eus396

[29] Shetty, A.K., Duckett, S.G., Ginks, M.R., Ma, Y., Sohal, M., Bostock, J., et al. (2013) Cardiac Magnetic ResonanceDerived Anatomy, Scar, and Dyssynchrony Fused with Fluoroscopy to Guide LV Lead Placement in Cardiac Resynchronization Therapy: A Comparison with Acute Haemodynamic Measures and Echocardiographic Reverse Remodelling. European Heart Journal, Cardiovascular Imaging, 14, 692-699. http://dx.doi.org/10.1093/ehjci/jes270

[30] Ypenburg, C., Schalij, M.J., Bleeker, G.B., Steendijk, P., Boersma, E., Dibbets-Schneider, P., et al. (2006) Impact of Viability and Scar Tissue on Response to Cardiac Resynchronization Therapy in Ischaemic Heart Failure Patients. European Heart Journal, 28, 33-41. http://dx.doi.org/10.1093/eurheartj/ehl379

[31] Reuter, S., Garrigue, S., Barold, S.S., Jaïs, P., Hocini, M., Haissaguerre, M., et al. (2002) Comparison of Characteristics in Responders versus Nonresponders with Biventricular Pacing for Drug-Resistant Congestive Heart Failure. American Journal of Cardiology, 89, 346-350. http://dx.doi.org/10.1016/S0002-9149(01)02240-8

[32] Friedman, D.J., Upadhyay, G.A., Altman, R.K., Orencole, M., Barrett, C.D., Mela, T., et al. (2013) The Anatomic and Electrical Location of the Left Ventricular Lead Predicts Ventricular Arrhythmia in Cardiac Resynchronization Therapy. Heart Rhythm, 10, 668-675. http://dx.doi.org/10.1016/j.hrthm.2012.12.025

[33] Ypenburg, C., van Bommel, R.J., Delgado, V., Mollema, S.A., Bleeker, G.B., Boersma, E., et al. (2008) Optimal Left Ventricular Lead Position Predicts Reverse Remodeling and Survival after Cardiac Resynchronization Therapy. Journal of the American College of Cardiology, 52, 1402-1409. http://dx.doi.org/10.1016/j.jacc.2008.06.046 
Scientific Research Publishing (SCIRP) is one of the largest Open Access journal publishers. It is currently publishing more than 200 open access, online, peer-reviewed journals covering a wide range of academic disciplines. SCIRP serves the worldwide academic communities and contributes to the progress and application of science with its publication.

Other selected journals from SCIRP are listed as below. Submit your manuscript to us via either submit@scirp.org or Online Submission Portal.
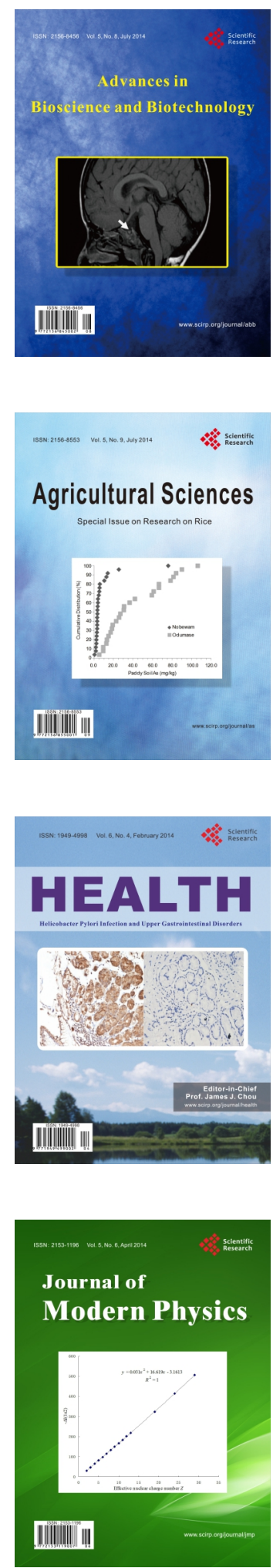
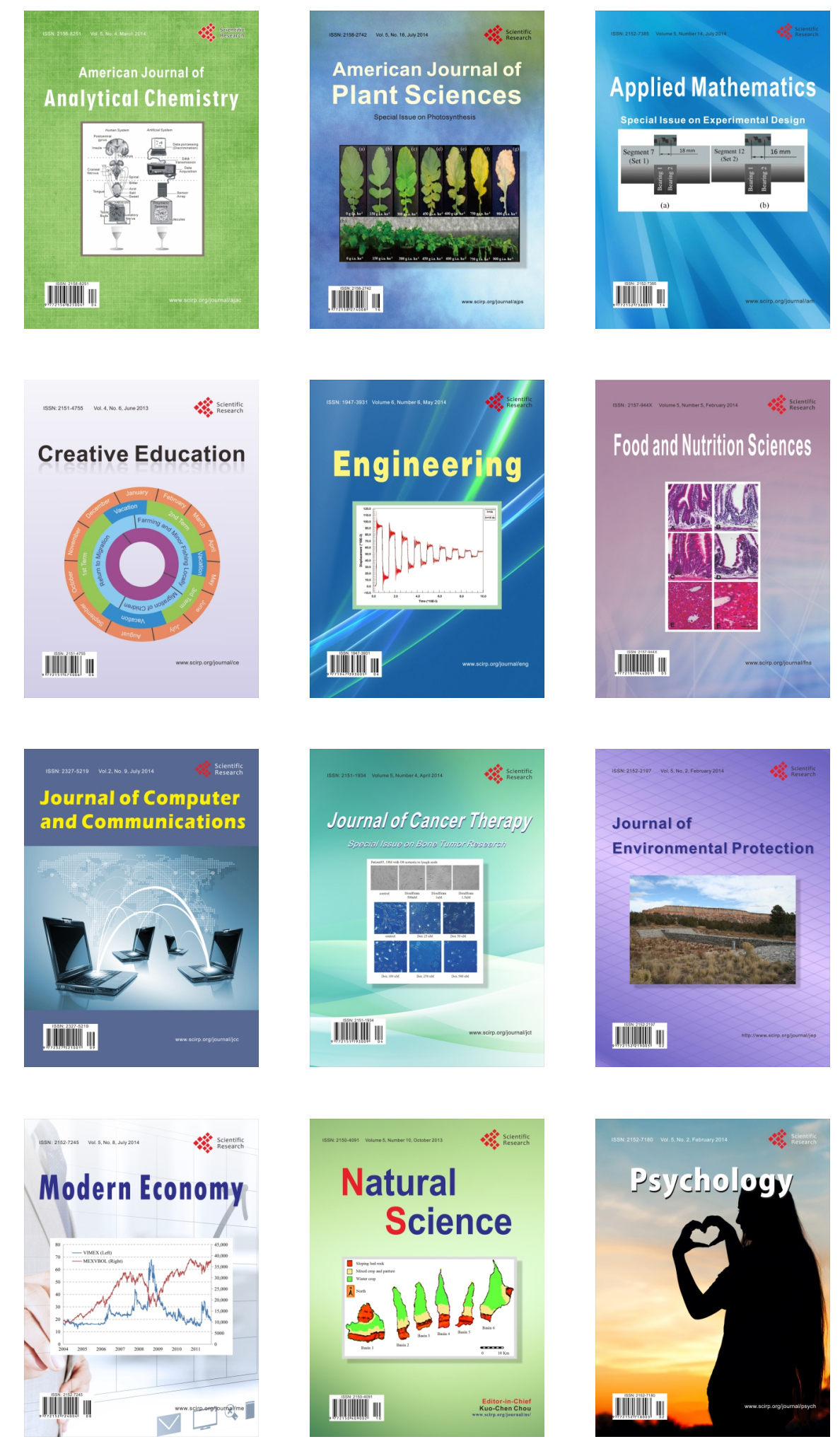